\title{
Características nutricionales de niños con parálisis cerebral. ARIE - Villa El Salvador, 2004
}

\author{
Aníbal Del Águila ${ }^{1}$, Patricia Áibar ${ }^{2}$
}

Resumen

Palabras clave

\begin{abstract}
Objetivos: Determinar la prevalencia y tipos de desnutrición en niños con parálisis cerebral (PC) e identificar problemas que conducen a ella. Diseño: Estudio clínico descriptivo. Lugar: Centrode Medicinay Rehabilitación Infantil ARIE, en el distrito de Villa El Salvador. Pacientes: Cincuentay tres niños con PC que asistían para su rehabilitación. Intervenciones: La información clínica fue obtenida por entrevista con la madre y para el diagnóstico nutricional se utilizó la clasificación de Waterlow. Para el diagnóstico de anemia, se evaluó los valores de hemoglobina y hematocrito. La enteroparasitosis fue diagnosticada por examen directo de heces y examen de Graham, en forma seriada. El procesamiento de información y análisis estadístico se realizó utilizando el programa SPSS 11.0. Principales medidas de resultados: Presencia de desnutrición, dificultades para alimentación, reflujo gastroesofágico, anemia y enteroparasitosis. Resultados: Se encontró desnutrición en $81,1 \%$ de niños, siendo la desnutrición crónica el diagnóstico más frecuente, en 43,5\%. Entre los problemas asociados a malnutrición, las dificultades para la alimentación estuvieron presentes en 94,3\% de los niños y síntomas de reflujo gastroesofágico, en 81,1\%. La prevalencia de anemia fue 32,4\% y de enteroparasitosis, 54,1\%. Conclusiones: Los niños con parálisis cerebral evaluados presentan una elevada prevalencia de desnutrición, relacionada al hallazgo frecuente de dificultades para la alimentación, reflujo gastroesofágicoyenteroparasitosis. Este grupo de niños constituye una población de riesgo alto de morbilidad y mortalidad.
\end{abstract}

Parálisis cerebral; desnutrición; reflujo gastroesofágico.
Nutritional characteristics of children with cerebral palsy. ARIE - Villa El Salvador, 2004

\section{Abstract}

Objective: To determine prevalence and types of malnutrition in children with cerebral palsy (CP) and to identify determinant problems. Design: Descriptive clinical study. Setting: Children's Medicine and Rehabilitation Centre ARIE, Villa El Salvador district. Patients: Fifty three children with CP receiving rehabilitation treatment. Interventions: Clinical information was obtained directly from the child's mother. Waterlow's classification was used for nutritional status evaluation. Anemia was determined by hemoglobin and hematocrit levels and intestinal parasitosis by feces examination and Graham's test. Data was processed with SPSS 11,0. Main outcome measures: Malnutrition, feeding problems, gastroesophageal reflux, anemia, intestinal parasitosis. Results: Prevalence of malnutrition was $81,1 \%$ and chronic malnutrition was the most frequent diagnosis, in 43,5\%. Disorders

Médico Pediatra, ARIE de Villa EI Salvador. Lima, Perú.

2 Médico Pediatra, Servicio de Pediatría del Hospital Angamos de EsSalud. Lima, Perú. associated with malnutrition like feeding problems were present in 94,3\% of children, and symptoms of gastroesophageal reflux disease in $81,1 \%$. Prevalence of anemia was $32,4 \%$ and intestinal parasitosis was present in $54,1 \%$ of children. Conclusions: Children with cerebral palsy had a high prevalence of malnutrition frequently associated to feeding difficulties, gastroesophageal reflux and intestinal parasitosis. These children represent a high-risk group for morbidity and mortality.

Key words: Cerebral palsy; malnutrition; gastroesophageal reflux.

\section{INTRODUCCIÓN}

La parálisis cerebral (PC) es la causa más frecuente de discapacidad en la población infantil $\left({ }^{1-4}\right)$. Este término es usado para describir el síndrome producido por lesión o daño del sistema nervioso central (SNC) durante períodos críticos de su desarrollo, con manifestaciones clínicas tan variables como cada niño que la presenta, pero que 
comparten en común el compromiso neurológico motor. La parálisis cerebral es "un término que cubre un grupo de síndromes de compromiso motor no progresivo, pero frecuentemente cambiante, secundario a lesiones o anormalidades del cerebro, ocurridas en estadios tempranos de su desarrollo" $\left({ }^{3}\right)$. Otros autores definen la parálisis cerebral infantil como "la secuela de una afección encefálica caracterizada por un trastorno persistente, pero no invariable, del tono, la postura y el movimiento, que aparece en la primera infancia y no solo es directamente secundaria a esta lesión no evolutiva del encéfalo, sino que se debe también a la influencia que dicha lesión ejerce en la maduración neurológica" $\left({ }^{5}\right)$. La lesión al encéfalo compromete la maduración neurológica de varias funciones, y no de una sola, lo que explica los múltiples compromisos que presenta el niño con PC, además del compromiso motor.

No existe mucha información sobre su incidencia en países en desarrollo $\left({ }^{4,6,7}\right)$. En países desarrollados, la incidencia de PC es 1,5 a 3 por 1000 recién nacidos $\left({ }^{3,8-11}\right)$. A nivel mundial, los avances en los cuidados obstétricos y perinatales en la últimas décadas y el surgimiento de las unidades de cuidados intensivos neonatales no se han acompañado de una disminución en la incidencia de niños con PC $\left({ }^{8,12,13}\right)$. Por el contrario, la incidencia de PC por 1000 recién nacidos se ha incrementado en el grupo de niños con peso bajo al nacer, lo cual al parecer es consecuencia del incremento sustancial en la supervivencia de este grupo de niños $\left({ }^{8,13}\right)$. Este riesgo aumenta a medida que el peso al nacimiento es menor $\left(^{1}\right)$. En un estudio hecho en Europa durante las décadas 1980-1990, la proporción de niños con PC en niños con peso al nacer menos de $1500 \mathrm{~g}$ fue 70 veces superior, comparado con aquellos con peso igual o superior a $2500 \mathrm{~g}$ al nacimiento $\left({ }^{11}\right)$. Las evidencias últimas sugieren que, contrariamente a lo que se pensaba, en aproximadamen- te 70 a $80 \%$ de casos la causa de PC se produce en el período prenatal, durante la gestación, mientras que la proporción de casos atribuidos a eventos perinatales, como la asfixia al nacimiento, es pequeña, aproximadamente $20 \%\left({ }^{12,13}\right)$.

Sus manifestaciones clínicas dependen fundamentalmente de la importancia de la extensión de la lesión, su localización, alteración funcional, del período en que ésta se produce, etc. por lo que no hay dos niños que estén afectados de la misma manera. Entre los desórdenes asociados, producidos por el daño cerebral que ocasiona la $\mathrm{PC}$, se encuentran retardo mental, dificultades para el aprendizaje, desorden de hiperactividad y déficit de atención, epilepsia, trastornos visuales, especialmente estrabismo, sordera, retardo del lenguaje, alteraciones de la percepción (agnosia y apraxia), disquinesias, entre otros $(2,8,14)$. Aunque no todos los niños con PC son afectados por estos problemas, éstos pueden tener un mayor impacto en la vida del niño que el mismo compromiso motor $\left(^{(2,14)}\right.$.

La clasificación de parálisis cerebral se basa en el tipo de falla motora y su localización. Se reconoce generalmente 5 tipos, según la falla motora: espástica, atetósica, atáxica, hipotónica, distónica o mixta. Según la localización de la falla motora o de las extremidades comprometidas: cuadripléjica, hemipléjica, parapléjica, dipléjica.

Entre los trastornos asociados al compromiso motor en el niño con PC, se encuentran dificultades para la alimentación o trastornos alimentarios. La lesión neurológica altera la función neuromuscular de forma directa o indirecta, causando disfunción motora oral, disquinesia faringoesofágica y dismotilidad esofágica e intestinal, que ocasionan dificultad para la apertura bucal, incoordinación en la succión, masticación, deglución, reflujo gastroesofágico, constipación, entre otros $\left({ }^{15,16}\right)$. Estos trastornos impiden que el niño pueda comer y beber 
adecuadamente, con una pobre ingesta calórica, lo cual ocasiona desnutrición y ésta a su vez produce un daño adicional al SNC $\left({ }^{17-20}\right)$.

La desnutrición es un trastorno sistémico que compromete a todos lo órganos y sistemas, estableciendo un estado metabólico que se adapta para sobrevivir a la falta crónica de alimentos $\left({ }^{21}\right)$. Sin embargo, el mayor efecto es el que ocurre en el desarrollo del SNC. Cuando la desnutrición se presenta durante la gestación o en edad posnatal temprana, el daño que produce en el cerebro puede ser irreversible $\left({ }^{22-27}\right)$.

La desnutrición durante el periodo de crecimiento cerebral, que es muy importante hasta los 3 años de vida, afecta la división celular, la mielinización y la sinaptogénesis, durante períodos críticos de su desarrollo ${ }^{(25)}$. Todas las regiones del cerebro son vulnerables, pero los periodos de vulnerabilidad varían dependiendo del grado máximo de división celular en una región en particular, de modo que la desnutrición podría afectar varias regiones cerebrales durante estos periodos $\left({ }^{23}\right)$. El peso del cerebro es menor en niños que mueren con desnutrición severa. En Bombay, el déficit medio de peso encefálico en niños malnutridos en comparación con los niños normales fue de $19 \%\left({ }^{28}\right)$. Este déficit del peso del cerebro va acompañado de un déficit del ADN total, es decir, del número total de neuronas y la circunferencia cefálica es inferior comparado con la de niños normales de la misma edad $\left({ }^{28}\right)$. Un hallazgo importante desde el punto de vista funcional es la reducción de la densidad de las sinapsis neuronales. Se ha comprobado una menor proporción de sinapsis por neurona en determinadas regiones del cerebro, como consecuencia de malnutrición posnatal temprana. La desnutrición precoz provoca alteraciones morfológicas y metabólicas en estructuras cerebrales que cumplen un rol fundamental en funciones cerebrales superiores, lo que produce pobre desarrollo intelectual, menor ren- dimiento escolar, pobre integración neurosensorial, menor memoria visual, menor rendimiento verbal, menores puntuaciones en pruebas de función motora, diseño de bloques, memoria para el diseño y aprendizaje incidental $\left({ }^{26-30}\right)$. El niño con desnutrición presenta cambios electroencefalográficos durante al menos un año después del episodio agudo $\left({ }^{28}\right)$. Existe en la actualidad consistente evidencia que los niños con retardo del crecimiento tienen deficiencias cognitivas $\left({ }^{31}\right)$. Los niños con desnutrición leve o moderada estudiados por varios investigadores presentan disminución de su interacción social, son menos activos y exploran menos que los niños bien nutridos ${ }^{\left({ }^{2}\right)}$, lo cual contribuye al retraso de su desarrollo. La recuperación de las funciones neurológicas parece ser posible sólo si el estado nutricional es mejorado antes de finalizar el periodo vulnerable de crecimiento $\left({ }^{23}\right)$.

Aunque la relación entre PC y desnutrición proteico-calórica es conocida $\left({ }^{15,20,33-36}\right)$, se tiene poca información de la realidad de esta relación en países en desarrollo $\left({ }^{17,35}\right)$, donde se añade el contexto de pobreza en que vive cerca de la mitad de la población, lo que implica no solo poca ingesta alimentaria sino también un escenario de alta vulnerabilidad de las familias que tienen que enfrentar el problema de tener un miembro con esta condición. La pobreza plantea un conjunto de limitaciones (ingresos bajos, falta de alimentos, falta de vivienda, falta de empleo, poco acceso a servicios de salud, saneamiento ambiental pobre, falta de educación, aumento de enfermedades infecciosas, etc.), que disminuyen la capacidad de una respuesta adecuada de estas familias. Esta realidad, hace a los niños con PC más vulnerables a la aparición de complicaciones, como la desnutrición y trastornos relacionados, todo lo cual incide directamente sobre su salud y calidad de vida.

El Centro de Medicina y Rehabilitación Infantil ARIE (Asociación para la Rehabilitación del Infante Excepcional), ubicado 
en el distrito de Villa El Salvador, atiende, a través de diferentes especialidades, a niños con discapacidad, siendo un grupo importante aquellos con PC. Su área de influencia es la población del cono Sur de Lima, especialmente de Villa El Salvador. Este distrito esta ubicado en la costa central, Departamento de Lima, a $20 \mathrm{~km}$ al sur del área central de Lima Metropolitana. Se ubica a 175 metros sobre el nivel del mar. Cuenta con una extensión de $35460 \mathrm{~km}^{2}$, que está dividida en cuatro áreas: urbano (56\% del área total del distrito), industrial, agropecuario y de playas. Cuenta con una población total de 344657 habitantes, de los cuales 92139 son menores de 14 años $(26,7 \%)$, correspondiendo a los menores de 5 años un total de 30098 niños $(8,7 \%)$. Más de $50 \%$ de la población del distrito vive en condiciones de pobreza y de extrema pobreza $\left({ }^{37}\right)$. Las dos primeras causas de morbilidad en la población infantil la constituyen las infecciones agudas de las vías respiratorias superiores e inferiores y las enfermedades infecciosas intestinales.

El objetivo del presente estudio es describir el estado nutricional de los niños con PC que asisten al Centro de Medicina y Rehabilitación Infantil ARIE y los trastornos asociados que pueden influir en dicho estado nutricional.

\section{MATERIALES Y MÉTODOS}

Todos los niños con PC que asisten para su rehabilitación al Centro de Medicina y Rehabilitación Infantil ARIE en el distrito de Villa El Salvador fueron evaluados para el estudio. No se incluyó aquellos con cardiopatía congénita o adquirida o con enfermedades pulmonares crónicas, tales como asma o fibrosis quística.

Los datos clínicos, antropométricos y de laboratorio de cada uno de los niños fueron registrados en una ficha clínica elaborada para tal fin. La información referida a los antecedentes fisiológicos, patológicos y familiares, trastornos alimentarios y signos y síntomas relacionados a reflujo gastroesofágico fue obtenida mediante entrevista con la madre o la persona a cargo del niño.

Los datos antropométricos fueron obtenidos directamente por los investigadores. La talla en los menores de 2 años fue obtenida con el tallímetro de lactantes y en los mayores de 2 años con el tallímetro de pie. Aquellos que no pudieron mantenerse de pie, fueron pesados junto con su madre en la balanza de pie, restándole posteriormente el peso de la madre. En todos los niños se obtuvo la longitud de la tibia (LT) del miembro inferior derecho, para calcular la talla, especialmente en niños con contracturas, espasticidad severa, deformidades esqueléticas o incapacidad para mantenerse de pie. En este caso, la talla se obtuvo aplicando la fórmula $(3,26 \times \mathrm{LT})+$ $30,8 \pm 1,4{ }^{(38)}$.

La evaluación nutricional se realizó a través de la combinación de los indicadores antropométricos talla para la edad (T/E) y peso para la talla $(\mathrm{P} / \mathrm{T})$, según la clasificación de Waterlow: normal, desnutrición aguda, desnutrición crónica o retardo en el crecimiento y desnutrición crónica reagudizada. Para el análisis de los indicadores, se utilizó los valores de referencia del National Center of Health Statistics (NCHS) de los EEUU. Se solicitó los siguientes exámenes auxiliares: hemoglobina y hematocrito, examen seriado (3 muestras) de heces para investigación de enteroparásitos y examen de Graham (2 muestras) para investigación de Enterobius vermicularis. Se realizó el procesamiento de la información y el análisis estadístico de tipo descriptivo utilizando el programa SPSS 11.

\section{RESULTADOS}

De 65 niños con parálisis cerebral evaluados, entre marzo y diciembre de 2004, 
53 completaron la evaluación y son los que forman parte de este estudio. De estos 53 niños, en 37 se obtuvo los exámenes auxiliares requeridos.

El mayor porcentaje de niños fue el del grupo de 6 a 10 años $(39,6 \%)$, seguido por el de 3 a 5 años (34\%), con edades que variaron entre 8 meses 20 días y 17 años 9 meses de edad, con ligero predominio del sexo masculino.

El $52,8 \%(n=28)$ de los niños fue producto de una primera gestación. Según la procedencia, la mayoría de niños $(54,7 \%)$ procedía del distrito de Villa El Salvador. El resto procedía de los distritos de Villa María del Triunfo, Chorrillos, Mala, Lurín.

Del total de niños, dieciséis $(30,2 \%)$ tenían epilepsia, de los cuales el $65 \%$ tomaban o habían tomado 2 o más anticonvulsivantes, simultáneamente. En relación al estado nutricional, el $81,1 \%$ de los casos $(n=43)$ tenía desnutrición, siendo la forma más frecuente la desnutrición crónica, que corresponde a $43,5 \%$ del total de niños desnutridos (Tabla 1).

Tabla 1. Distribución de los 53 niños con PC de acuerdo al estado nutricional, según la clasificación de Waterlow.

\begin{tabular}{lrr}
\hline \multicolumn{1}{c}{ Diagnóstico del estado nutricional } & Número & $\%$ \\
\hline 1) Normal & 10 & 18,9 \\
2) Desnutrición & 43 & 81,1 \\
- Desnutrición aguda & 3 & 5,7 \\
Desnutrición aguda de primer grado & 1 & 1,9 \\
Desnutrición aguda de segundo grado & 2 & 3,8 \\
Desnutrición aguda detercer grado & 0 & 0 \\
- Desnutrición crónica & 23 & 43,5 \\
Desnutrición crónica de primer grado & 11 & 20,8 \\
Desnutrición crónica de segundo grado & 9 & 17,0 \\
Desnutrición crónica de tercer grado & 3 & 5,7 \\
- Desnutrición crónica reagudizada & 17 & 32,1 \\
Desnutrición crónica reagudizada de primer grado & 11 & 20,8 \\
Desnutrición crónica reagudizada de segundo grado & 2 & 3,8 \\
Desnutrición crónica reagudizada de tercer grado & 4 & 7,5 \\
Total & 53 & 100 \\
& & \\
\hline
\end{tabular}

Cinco niños con desnutrición crónica tenían además peso para la talla superior al percentil 95, uno $(1,9 \%)$ con sobrepeso y cuatro $(7,5 \%)$ con obesidad. El grupo de edad con mayor porcentaje de desnutrición fue el de 6 a 10 años, con $95 \%$, seguido por el de 3 a 5 años, con $77,8 \%$.

En cuanto a trastornos asociados, la mayoría de niños evaluados tenía dificultades para la alimentación, 94,3\%, y síntomas asociados a reflujo gastroesofágico, $81,1 \%$ (Tabla 2).

Tabla 2. Distribución de casos de niños con PC por grupo de edad con trastornos de la alimentación y síntomas asociados a reflujo gastroesofágico.

\begin{tabular}{|c|c|c|c|c|c|}
\hline \multirow[t]{2}{*}{ Grupo de edad } & \multirow{2}{*}{$\begin{array}{l}\text { Número } \\
\text { de niños } \\
\text { (N) }\end{array}$} & \multicolumn{2}{|c|}{$\begin{array}{l}\text { Trastornos } \\
\text { alimentarios }\end{array}$} & \multicolumn{2}{|c|}{$\begin{array}{l}\text { Síntomas asociados a } \\
\text { reflujo gastroesofágico }\end{array}$} \\
\hline & & Nro. & $\%$ de $\mathrm{N}$ & Nro. & $\%$ de $\mathrm{N}$ \\
\hline $0-11$ meses & 2 & 2 & 100 & 1 & 50 \\
\hline 1 - 2 años & 8 & 7 & 87,5 & 8 & 100 \\
\hline 3 - 5 años & 18 & 17 & 94,4 & 13 & 72,2 \\
\hline $6-10$ años & 21 & 20 & 95,2 & 18 & 85,7 \\
\hline Más de 10 años & 4 & 4 & 100 & 3 & 75 \\
\hline Total & 53 & 50 & 94,3 & 43 & 81,1 \\
\hline
\end{tabular}

Los trastornos alimentarios más frecuentes fueron sialorrea, dificultad para la masticación e incoordinación motora o falta de control cefálico (Tabla 3). El 81,2\% de los niños tenía entre 2 y 6 trastornos alimentarios.

Asimismo, se encontró la presencia de constipación en treinta niños $(56,6 \%)$. En cuanto a las características alimentarias, la mayoría de niños necesitaba ayuda para alimentarse, $77,4 \%$, e ingería dieta licuada o triturada, $51 \%$. El $71,7 \%$ de niños recibía de 2 a 3 comidas por día.

Entre los síntomas asociados a RGE más frecuentes, estuvieron las infecciones respiratorias recurrentes, sibilancias y regurgitación y/o vómito posprandial (Tabla 4). 
Tabla 3. Frecuencia de trastornos alimentarios.

\begin{tabular}{lcr}
\hline \multicolumn{1}{c}{ Trastornos alimentarios } & Número & $\%$ \\
\hline Sialorrea & 41 & 77,4 \\
Dificultad para la masticación & 35 & 66 \\
Incoordinación motora o falta & & \\
de control cefálico & 24 & 45,3 \\
Regurgitación y/o vómitos posprandiales & 21 & 39,6 \\
Dificultad para la deglución & 18 & 34 \\
Dificultad para la succión & 13 & 24,5 \\
Lengua hipertónica & 12 & 22,6 \\
Apertura disminuida de la boca & 11 & 20,8 \\
Falta de cierre de labios alrededor & & \\
de la cuchara & 9 & 17 \\
Rechazo a la comida & 7 & 13,2 \\
\hline
\end{tabular}

Se encontró, además, que el número de trastornos alimentarios y el de síntomas asociados a reflujo gastroesofágico fue mayor en los niños con desnutrición que en los niños con estado nutricional normal. De los 37 niños en los que se obtuvo los resultados de hemoglobina y hematocrito, 12 $(32,4 \%)$ tenían anemia. De los 37 niños con resultados de examen de heces, $54,1 \%$ tenía enteroparasitosis, siendo las más frecuentes Enterobius vermiculares, Giardia lamblia y Ascaris lumbricoides.

\section{DISCUSIÓN}

Destaca en este estudio la elevada prevalencia de desnutrición en el grupo de niños evaluados, $81,1 \%$ (Tabla 1), muy superior al encontrado en otros trabajos $\left.{ }^{7,15,33,35,39-42}\right)$. El tipo de desnutrición más frecuente fue la desnutrición crónica, como ocurre en el resto de la población infantil de nuestro país, siendo sustancialmente diferente el alto porcentaje de desnutrición crónica reagudizada $(32,1 \%)$ encontrado en estos niños (Tabla 1). Ésta es una forma severa de desnutrición en la clasificación de Waterlow, que significa que al retardo de crecimiento producido por la falta crónica de alimentos o por la suma de varios episodios agudos de desnutrición se agrega un proceso de desnutrición aguda con disminución del peso para la talla, que se conoce como emaciación. Esto produce un delicado estado de salud, con un estado metabólico alterado y disminución de la inmunidad, que lo hace susceptible a complicaciones infecciosas, la mayor causa de muerte en estos niños $\left({ }^{43,44,45}\right)$. La incidencia, prevalencia y gravedad de las infecciones son mayores en los niños con malnutrición grave, en quienes la invasión y multiplicación de los agentes patógenos es mayor y las tasas de morbilidad son altas $\left({ }^{46}\right)$.

La desnutrición es un trastorno que compromete a todos lo órganos y sistemas, estableciendo un estado metabólico que se adapta para sobrevivir a la falta crónica de alimentos $\left({ }^{21}\right)$. Sin embargo, el mayor efecto es el que ocurre en el desarrollo del SNC. Cuando la desnutrición se presenta durante la gestación o en edad posnatal temprana, el daño que produce en el SNC es irreversible $\left(^{22-26}\right)$. La desnutrición en niños con PC en edades tempranas que no es tratada a tiempo agrega un daño adicional al SNC, lo que empeoraría la condición neurológica, restándole posibilidades de una mayor recuperación.

La elevada prevalencia de desnutrición encontrada se diferencia de otras revisio-

Tabla 4. Frecuencia de síntomas asociados a reflujo gastroesofágico.

\begin{tabular}{lrr}
\hline $\begin{array}{l}\text { Síntomas asociados a } \\
\text { reflujo gastroesofágico }\end{array}$ & Número & $\%$ \\
\hline Infecciones respiratorias recurrentes & 34 & 64,2 \\
Sibilancias & 25 & 47,2 \\
Regurgitación y/o vómito posprandial & 20 & 37,7 \\
Tos crónica & 18 & 34 \\
Tos durante o después de la alimentación & 18 & 34 \\
Distonía extensora durante la alimentación & 13 & 24,5 \\
Estridor recurrente & 11 & 20,8 \\
Llanto durante o después de la alimentación & 6 & 11,3 \\
\hline
\end{tabular}


nes, la mayoría hecha en países desarrollados, por el contexto de pobreza en que vive la población estudiada. Esta situación no solo implica una pobre alimentación en cantidad y calidad, sino un conjunto de limitaciones; éstas disminuyen la capacidad de una respuesta adecuada a los problemas que trae el tener un miembro de la familia con esta condición. Por estas razones, el grupo de niños con PC estudiados constituye una población altamente vulnerable a morbilidad y mortalidad.

El grupo con mayor porcentaje de desnutrición fue el de 6 a 10 años de edad, $90,5 \%$, seguido por el grupo de 3 a 5 años, con $77,8 \%$. El mayor porcentaje de desnutrición se observa en los niños con mayor edad, debido a que la desnutrición crónica se va estableciendo por la suma de varios episodios de desnutrición aguda o por la falta crónica de alimentos; ello se refleja en una talla baja para la edad, que se hace más evidente conforme mayor sea la edad. El hecho de que en 5 niños, 9,4\%, con desnutrición crónica encontráramos sobrepeso y obesidad, es decir exceso de peso para la talla, refleja la mala calidad de alimentación, con una excesiva ingesta de calorías sobre la de proteínas, que no mejora su estado de salud; por el contrario, va en perjuicio de ella $\left({ }^{43}\right)$.

En nuestro trabajo, el porcentaje de niños con trastornos alimentarios es de 94,3\% (Tabla 2), porcentaje superior también al que comunican otras revisiones $\left({ }^{15,47,48}\right)$. Se observó que, mientras mayor es el número de trastornos alimentarios mayor es el porcentaje de niños con desnutrición y que el promedio del número de trastornos alimentarios es mayor en los niños con desnutrición que en los niños con estado nutricional normal, especialmente en las formas más graves de desnutrición, como son la desnutrición crónica de tercer grado y la desnutrición crónica reagudizada de tercer grado, lo que reafirma la relación entre dificultades para la alimentación y desnutrición en niños con PC $\left({ }^{17-20}\right)$. El porcentaje de niños con trastornos alimentarios es similar en todos los grupos de edad (Tabla 2), lo que hace suponer que estos trastornos se presentan desde temprana edad y permanecen en edades posteriores. Es importante señalar que, las dificultades severas para la alimentación pueden preceder al diagnóstico de $\mathrm{PC}\left({ }^{48}\right)$.

Diferentes trabajos en niños con PC concluyen afirmando que, las dificultades para la alimentación representan un predictor importante de un mal estado de salud, expresado en deficiencias nutricionales y una mala calidad de vida $(19,47,49,50)$. Cuando las dificultades para alimentarse son tempranas, persistentes y severas, son marcadores de bajo crecimiento, deficiente volumen de alimentación y limitaciones en el desarrollo neuromotor $\left(^{51}\right)$. Se ha estudiado el impacto de los trastornos de la alimentación en el desarrollo que alcanzan los niños afectados por parálisis cerebral $\left({ }^{51}\right)$. La rehabilitación neurológica en niños con PC que a temprana edad reciben tratamiento de los trastornos de alimentación, obtienen mayores logros en la coordinación viso-motora y en el lenguaje que aquellos niños que no la reciben $\left({ }^{52}\right)$. Por estas razones, la rehabilitación de la función alimentaria debe ser parte integral del plan general de rehabilitación de los niños con PC y debe iniciarse tan pronto sea posible. Cuando la intervención nutricional se posterga más allá de los 8 años, se puede aún mejorar el peso, pero el crecimiento lineal es limitado $\left({ }^{15}\right)$.

Es importante señalar que $77,4 \%$ de niños no tiene la capacidad de alimentarse por sí solos. La capacidad de alimentarse por uno mismo requiere un desarrollo neurológico adecuado; es necesario coordinar los movimientos de succión, masticación y deglución con los movimientos respiratorios; se precisa de un control del esqueleto axial; deben moverse brazos $\mathrm{y}$ manos intencional y coordinamente, etc. $\left({ }^{16}\right)$. Este elevado porcentaje revela la seve- 
ridad del compromiso neurológico en estos niños.

En lo que se refiere a la consistencia de los alimentos, el mayor porcentaje de niños la recibe licuada o triturada, $51 \%$, que conlleva a una dieta hipocalórica que incide sobre su estado nutricional. En relación a la frecuencia de la alimentación, se encontró que la mayor proporción de niños $(71,7 \%)$ reciben 2 a 3 comidas al día. Lo que se recomienda en estos niños es mayor número de comidas al día en poco volumen, debido a la menor capacidad gástrica y al vaciamiento gástrico retardado.

El reflujo gastroesofágico (RGE) es común en niños con PC $\left({ }^{53,54,55}\right)$. En nuestro trabajo, la proporción de niños con síntomas asociados a RGE fue de $81,1 \%(n=43)$ (Tabla 2), siendo los síntomas más frecuentes las infecciones respiratorias recurrentes, sibilancias, regurgitación y/o vómito posprandial, tos crónica y tos durante o después de la alimentación (Tabla 4). Destaca en este sentido el que el síntoma más frecuente de RGE encontrado en estos niños sean las infecciones respiratorias y no los síntomas propiamente digestivos, lo que señala la importancia de evaluar si un niño con PC tiene RGE, para disminuir la posibilidad de complicaciones respiratorias que pueden ser fatales. Varios estudios informan diferentes porcentajes de RGE en niños con PC, que van desde $24 \%$ a $86 \%$, confirmados con exámenes, como monitorización por 24 horas del $\mathrm{pH}$ intraesofágico, gammagrafía, manometría esofágica, radiografía contrastada de esófago, etc. $\left({ }^{7,15,53-57)}\right.$. La gammagrafía dinámica de esófago realizada en uno de los niños demostró presencia de RGE y mediana probabilidad de aspiración bronquial (Figura 1).

El reflujo con aspiración es responsable de neumonía recurrente en niños con alteraciones neurológicas. Si el RGE es significativo y frecuente, se incrementa el riesgo de aspiración de alimentos y neumonía

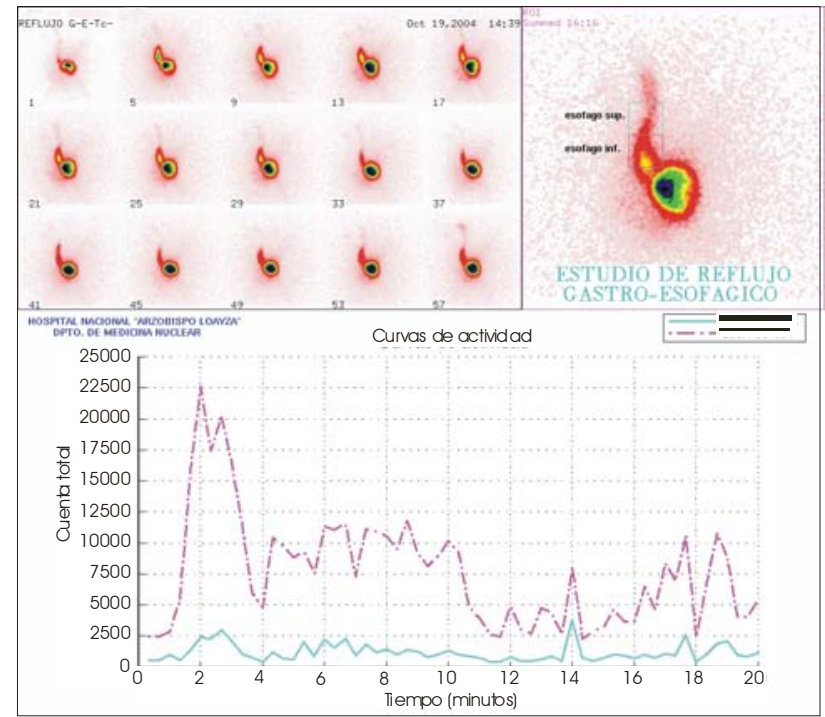

Figura 1. Imágenes de la gammagrafía realizada a niño de 1 año y 4 meses con parálisis cerebral, en las que se observa picos de ascenso de la sustancia con TC-99 hacia el esófago, luego de haber sido digerido hasta el estómago, lo cual comprueba la presencia del reflujo gastroesofágico.

por aspiración. Durante el estudio, uno de los niños falleció por bronconeumonía. La regurgitación, junto con la pobre ganancia de peso, es la presentación más común de RGE en lactantes, pero ocurre con menos frecuencia en niños mayores y adultos $\left({ }^{58}\right)$. Desde el punto de vista clínico, la presencia de tos durante la deglución es un indicador muy confiable de riesgo de aspiración, con sensibilidad de $74 \%$, especificidad de $71 \%$ y valor predictivo negativo de $77 \%\left({ }^{38}\right)$.

Se reconoce que la RGE es causa de desnutrición en niños con PC, debido a la ingesta calórica insuficiente por odinofagia que ocasiona rechazo a la alimentación y a la sustancial pérdida de nutrientes en los vómitos, además de la vacilación de los padres para alimentar a su niño, que constantemente regurgita sus alimentos $\left({ }^{58,59}\right)$. En nuestro trabajo se observa que, a mayor número de síntomas asociados a RGE que presenta el niño, mayor es el porcentaje de niños con desnutrición, y que el promedio 
de síntomas asociados a RGE es mayor en los niños con distintos tipos de desnutrición que en los niños con estado nutricional normal. Se observa que este promedio es el doble en los niños con desnutrición crónica de tercer grado, y casi el triple en niños con desnutrición crónica reagudizada de tercer grado. Si bien, esto no demuestra asociación alguna, apunta a señalar una tendencia a la desnutrición en los niños con mayor número de síntomas asociados a RGE. Otras complicaciones del RGE son aquellas secundarias a esofagitis (estenosis, anemia, esófago de Barrett y adenocarcinoma) y se desarrollan en el $10 \%$ de niños no tratados $\left({ }^{58}\right)$.

Un comentario aparte merece el hecho de que $52,8 \%$ de niños fue producto de una primera gestación, superior a lo que refieren otras publicaciones $\left({ }^{60}\right)$. Este hecho podría reflejar la falta de un control obstétrico y perinatal adecuado, en madres por lo demás jóvenes y, según refirieron en la encuesta, con buen estado de salud al iniciar la gestación. Esto implicaría que las condiciones de vida y la falta de acceso a los servicios de salud, por razones geográficas, económicas o culturales, de las gestantes podrían tener un rol en la incidencia de casos de PC.

De los 37 pacientes en que se obtuvo resultados de hemoglobina y hematocrito, 12 $(32,4 \%)$ tuvieron diagnóstico de anemia. Además de la falta de ingesta de hierro, la anemia, en este caso, puede ser causada por enteroparasitosis y por la pérdida de sangre que ocasiona la esofagitis debido al RGE. La importancia de este diagnóstico radica en que los efectos a largo plazo de anemia por deficiencia de hierro durante la niñez pueden ser permanentes, por alteraciones irreversibles en el desarrollo cerebral $\left({ }^{61}\right)$, lo que produciría mayor deterioro neurológico en el niño con PC. Estudios longitudinales indican consistentemente que, niños que fueron anémicos en la niñez temprana continúan con pobre desarrollo cognitivo y motor y menor rendimiento es- colar en edades más tardías $\left({ }^{62}\right)$. Hay que señalar que las bajas concentraciones de hemoglobina y el hematocrito bajo representan el tercer estadio de deficiencia de hierro, luego que se han depletado las reservas orgánicas, de manera que cuando se diagnostica anemia por los valores de hemoglobina y hematocrito la deficiencia de hierro ha venido ocurriendo semanas o meses previos, lo cual ya ocasiona daño en el SNC en desarrollo. Entonces, la anemia es una manifestación tardía de la deficiencia de hierro. En este caso, se habla de niños deficientes de hierro no anémicos. La deficiencia de hierro reflejada por la producción restringida de hemoglobina está asociada en forma significativa con retardo mental leve o moderado, y este riesgo se incrementa con la severidad de la anemia $\left.{ }^{(63}\right)$. La anemia por deficiencia de hierro está fuertemente asociada a estado socioeconómico bajo y pobreza, como en el caso de estos niños, y es más prevalente durante los 2 primeros años de vida, cuando el cerebro del niño esta aún en desarrollo. Los picos de captación de hierro en el cerebro ocurren durante el período de rápido crecimiento neuronal; más aún, el hierro intracelular está involucrado en la síntesis, captación y degradación de neurotransmisores que intervienen en el procesamiento de la información $\left({ }^{63}\right)$.

Otra patología asociada a condiciones de pobreza es la parasitosis intestinal. En nuestro trabajo, de los 37 niños en que se realizó exámenes de heces, 20 niños $(54,1 \%)$ tuvieron el diagnóstico de enteroparasitosis. Este diagnóstico es relevante por la asociación entre parasitosis intestinal y desnutrición. Existe información suficiente que indica que la infección parasitaria del tracto intestinal puede causar malnutrición $\left({ }^{44,46,64}\right)$. $\mathrm{Su}$ presencia afecta la digestión y absorción de nutrientes. Entre los mecanismos mediante los cuales los parásitos intestinales deterioran el estado nutricional, se encuentra secreción de toxinas que afectan la digestión, 
mala absorción de nutrientes a nivel de la membrana del enterocito, competencia entre el huésped y el parásito por los nutrientes, pérdida gastrointestinal de nutrientes y degradación de los nutrientes $\left({ }^{65}\right)$.

Los parásitos más frecuentemente encontrados han sido descritos como causantes o agravantes de malnutrición $\left({ }^{65-67}\right)$. En ambientes de pobreza, la presencia de Giardia es generalizada y casi todos los niños se infectan con este parásito durante el primer año de vida $\left({ }^{46}\right)$.

Por último, otro factor importante que incide en el estado nutricional, especialmente en niños que reciben varias medicaciones, como los niños con PC, es la interacción droga-nutriente. En el caso de estos niños, nos referimos particularmente a las drogas anticonvulsivantes. Del total de niños evaluados, 16 (30,2\%) tenían epilepsia y $65 \%$ de ellos tomaba o había tomado más de un anticonvulsivante, simultáneamente. Los diferentes anticonvulsivantes producen depleción de varios nutrientes, con el consiguiente deterioro del estado nutricional del niño $\left({ }^{38}\right)$ :

De manera que, la ingesta de medicamentos debiera ser tomada en cuenta al realizar la evaluación nutricional, para evitar o aminorar los efectos adversos sobre el estado nutricional.

Los resultados de este estudio nos llevan a coincidir con las recomendaciones del Comité de Nutrición de la Sociedad Pediátrica de Canadá, que señala que es inaceptable no tratar la desnutrición asociada a discapacidad del neurodesarrollo $\left({ }^{18}\right)$.

Nuestros hallazgos, en concordancia con la literatura señalada, resaltan la importancia de la evaluación y tratamiento del estado nutricional y de todos los problemas que influyen en éste en el niño con PC, en forma permanente, como parte integral de su tratamiento y rehabilitación.
En resumen, el grupo de niños con PC estudiado presenta una alta prevalencia de desnutrición $(81,1 \%)$, superior a lo encontrado en la literatura revisada, en la que destaca el alto número de niños con desnutrición crónica $(43,5 \%)$ y desnutrición crónica reagudizada $(32,1 \%)$. Los trastornos inherentes a la condición de PC, como trastornos alimentarios $(94,3 \%)$, reflujo gastroesofágico $(81,1 \%)$, y los trastornos asociados a la condición socioeconómica, como anemia $(32,4 \%)$ y enteroparasitosis $(54,05 \%)$, explicarían la elevada prevalencia de desnutrición en los niños evaluados. La prevalencia de desnutrición y de trastornos asociados descritos en este trabajo, junto con la pobreza, determinan que este grupo de niños constituya una población altamente vulnerable a morbilidad y mortalidad.

\section{REFERENCIAS BIBLIOGRÁFICAS}

1. Blanco MA. Síndrome de parálisis cerebral. En: Enfermedades invalidantes de la infancia: un enfoque integral de rehabilitación. Santiago: Sociedad Pro Ayuda del Niño Lisiado; 1995.

2. Centro Argentino de Restauración Neurológica [homepage on the Internet]. Buenos Aires: Instituto CAREN; c19952006 [citado el 19 de abril de 2004]. Disponible en: http:// www.neurorehabilitacion.com/default.htm

3. Rosenbaum P. Cerebral palsy: what parents and doctors want to know. A clinical review. British Medical Journal. 2003;326:970-4.

4. Larguía A. Consenso argentino sobre parálisis cerebral. Rol del cuidado perinatal. Arch Argent Pediatr. 2000;98(4):2537.

5. Espacio Logopédico.com [homepage on the Internet]. Barcelona: Majo Producciones; c2001-2006 [citado el 17 de mayo de 2004]. Disponible en: http:// www.espaciologopedico.com

6. Magally S. Hay en México 500 mil personas con parálisis cerebral. México, DF: agencia de noticias de CIMAC [citado el 19 de abril de 2004]. Disponible en: http:// www.cimacnoticias.com

7. Nardi A. Estado nutricional y factores condicionantes en niños con parálisis cerebral infantil. Hospital Central «Antonio Maria Pineda». Trabajo presentado para optar al título de Especialista en Puericultura y Pediatría. Universidad Centroccidental Lisandro Alvarado. Decanato de Medicina. Barquisimeto, Venezuela. Febrero, 2001. 
8. Kuban KCK, Leviton A. Cerebral palsy. N Engl J Med. 1994;330:188-95.

9. Surveillance of cerebral palsy in Europe. A collaboration of cerebral palsy surveys and registers. Surveillance of cerebral palsy in Europe (SCPE). Dev Med Child Neurol. 2000;42(12):816-24.

10. Winter S, Autry A, Boyle C, Yeargin-Allsopp M. Trends in prevalence of cerebral palsy in a population-based study. Pediatrics. 2002;110(6):1220-5.

11. Jonson A. Prevalence and characteristics of children with cerebral palsy in Europe. Developmental Medicine and Child Neurology. 2002;44:633-40.

12. Perlman JM. Intrapartum hypoxic-ischemic cerebral injury and subsequent cerebral palsy: Medicolegal issues. Pediatrics. 1997;99(6):851-9.

13. Torfs CP, van den Berg BJ, Oechsli FW, Cummins S. Prenatal and perinatal factors in the etiology of cerebral palsy. Journal of Pediatrics. 1990;116:615-9.

14. Behrman R, Kliegman R, Jenson H (eds). Nelson Textbook of Pediatrics. $16^{\text {th }}$ edition. Philadelphia: WB Saunders Company; 2000: 1843-5.

15. Schwarz SM, Corredor J, Fisher-Medina J, Cohen J, Rabinowitz S. Diagnosis and treatment of feeding disorders in children with developmental disabilities. Pediatrics. 2001;108(3):671-6.

16. Moreno JM, Galiano MJ, Valero MA, León M. Alimentación en el paciente con parálisis cerebral. Acta Pediatr Esp. 2001;59(1):17-25.

17. Gangil A, Patwari A, Aneja S, Ahuja B, Anand VK. Feeding problems in children with cerebral palsy. Indian Pediatrics. 2001;38:639-46.

18. Nutrition Committee of the Canadian Paediatric Society. Undernutrition in children with a neurodevelopmental disability. Canadian Medical Association Journal. 1994;151(6):753-9. Reaffirmed March 2004.

19. Fung EB, Samson-Fang L, Stallings VA, Conaway M, Liptak $\mathrm{G}$, Henderson RC, et al. Feeding dysfunction is associated with poor growth and health status in children with cerebral palsy. J Am Diet Assoc. 2002;102(3):361-73.

20. Stallings VA, Cronk CE, Zemel BS, Charney EB. Body composition in children with spastic quadriplejic cerebral palsy. J Pediatr. 1995;126(5 Pt 1):833-9.

21. Coward WA, Lunn PG. The biochemistry and physiology of kwashiorkor and marasmus. British Medical Bulletin. 1981;37(4):19-24.

22. Hesse H. La desnutrición proteico-calórica y el sistema nervioso [monografía en Internet]. Barcelona: Societat Catalana de Neurología [citado el 17 de enero de 2005]. Disponible en: http://www.scn.es/cursos/tropical/DESNUTRICION.htm

23. Winick M, Morgan BLG. Nutrition and brain development. En: Walker WA, Watkins JB (eds). Nutrition in Pediatrics. Boston/Toronto: Little Brown and company; 1985.

24. Carnegie Task Force on Meeting the Needs of Our Youngest Children. Starting Points: Meeting the Needs of Our Youngest Children. New York: Carnegie Corporation of New York; 1994. p. 5.
25. Marcondes A, Cavallo A, Gazali S, Machado D, Léfevre A, Martins Campos V, Quarentei G, Barbieri D. Estudios neuropsicomotores en la desnutrición. I. Aspectos de la recuperación bioquímica y neuropsicomotora, con especial interés en las alteraciones neumoencefalográficas. Mesa Redonda Nro. 5. Nutrición y desarrollo mental. XII Congreso Internacional de Pediatría. México D.F. 1963.

26. Nelson M. Vitamin and mineral supplementation and academic performance in school children. Proc Nutr Soc. 1992;51:30313.

27. Pollitt E. Developmental sequel from early nutritional deficiencies: Conclusive and probability judgements. J Nutr. 2000;130:350S-3S.

28. Waterlow JC. Efectos de la MPE sobre la estructura y las funciones de los órganos. En: Waterlow JC (ed). Malnutrición proteico-energética. Publicación Científica Nro. 555. Washington DC: Organización Panamericana de la Salud; 1996.

29. Grantham-McGregor SM. Efecto de la malnutrición en el desarrollo mental. En: Waterlow JC. Malnutrición proteicoenergética. Publicación Científica Nro. 555. Washington DC: Organización Panamericana de la Salud; 1996.

30. Leiva B, Inzunza N, Pérez H, Castro V, Jansana JM, Toro $\mathrm{T}$, y col. Algunas consideraciones sobre el impacto de la desnutrición en el desarrollo cerebral, inteligencia y rendimiento escolar. ALAN. 2001;51(1):64-71

31. Grantham-McGregor SM. Linear growth retardation and cognition. Lancet. 2002;359:542.

32. Graves PL. Nutrition, infant behavior and maternal characteristics: a pilot study in West Bengala, India. American Journal of Clinical Nutrition. 1976,29:305-19.

33. Dahl M, Thommessen M, Rasmussen M, Selberg. Feeding and nutritional characteristics in children with moderate or severe cerebral palsy. Acta Paediatric. 1996;85:697-701.

34. Socrates C, Grantham-McGregor SM, Harknett SG, Seal AJ. Poor nutrition is a serious problem in children with cerebral palsy in Palawan, the Philippines. Int J Rehabil Res. 2000;23(3):177-84.

35. Samson-Fang L, Fung E, Stallings V, Conaway M, Worley G, Rosenbaum P, et al. Relationship of nutritional status to health and societal participation in children with cerebral palsy. J Pediatr. 2002;141(5):637-43.

36. Krick J, Murphy PE, Markham JF, Shapiro BK. Body composition in children with spastic quadriplegic cerebral palsy. J Pediatr. 1995;126(5 Pt 1):833-9.

37. Ministerio de Salud del Perú, Red de Servicios de Salud de Villa El Salvador, Unidad de Estadísticas. Datos estadísticos 2003 - 2004. Lima: MINSA; 2005.

38. Mariño M, Rojas Y. Manejo nutricional de patologías neurológicas. III Curso de manejo nutricional de patologías pediátricas. Caracas 26 de Abril de 2002.

39. Hung JW, Hsu TJ, Wu PC, Leong CP. Risk factors of undernutrition in children with spastic cerebral palsy. Chang Gung Med J. 2003;26(6):425-32.

40. Troughton KE, Hill AE. Relation between objectively measured feeding competence and nutrition in children with 
cerebral palsy. Dev Med Child Neurol. 2001;43(3):18790.

41. Vik T, Skrove MS, Dollner H, Helland G. Feeding problems and growth disorders among children with cerebral palsy in south and north Trondelag. Tidsskr Nor Laegeforen. 2001;121(13):1570-4.

42. Zulueta D, Romero M, Toledo E, Ferrer N. Patrones de alimentación y evaluación nutricional en niños deshabilitados. Rev Cubana Salud Pública. 2003;29(2):111-16.

43. Ranjit Kumar Chandra. Nutrition and the immune system. Symposium on nutrition and immunity in serious illness. Proceedings of the Nutrition Society. 1993;52:77-84.

44. Berkowitz F. Infections in children with severe protein-energy malnutrition. Pediatr Infect Dis J. 1992;11(9):750-9.

45. Shankuntla Puri, Ranjit Kumar Chandra. Malnutrition and immunity. En: Grand RJ, Sutphen JL, Dietz WH. Pediatric Nutrition. Theory and Practice. Boston, MA: Butterworths Publishers; 1987.

46. Waterlow JC. Nutrición e infección. En: Waterlow JC (ed). Malnutrición proteico-energética. Publicación Científica Nro. 555. Washington DC: Organización Panamericana de la Salud; 1996.

47. Thommessen M, Kase BF, Riis G, Heiberg A. The impact of feeding problems on growth and energy intake in children with cerebral palsy. Eur J Clin Nutr. 1991;45(10):479-87.

48. Reilly S, Skuse D, Poblete X. Prevalence of feeding problems and oral motor dysfunction in children with cerebral palsy: a community survey. J Pediatr. 1996;129(6):877-82.

49. Liptak GS, O’Donnell M, Conaway M, Chumlea WC, Wolrey $\mathrm{G}$, et al. Health status of children with moderate to severe cerebral palsy. Dev Med Child Neurol. 2001,43(6):364-70.

50. Reilly S, Skuse D. Characteristics and management of feeding problems of young children with cerebral palsy. Dev Med Child Neurol. 1992;34(5):379-88.

51. Motion S, Northstone K, Emond A, Stucke S, Golding J. Early feeding problems in children with cerebral palsy: weight and neurodevelopmental outcomes. Dev Med Child Neurol. 2002;44(1):40-3.

52. Borkowska M. Effect of rehabilitation of the feeding function on the development of visual-motor coordination and speech in children with infantile cerebral palsy. Probl Med Wieku Rozwoj. 1979;9:209-34.

53. Mazzoleni S, Drigo P, Da Dalt L, Battistella PA, Casara Gl, Donzelli F, Zachello F. Gastroesophageal reflux and infantile cerebral palsy: our experience and review of the literature. Pediatr Med Chir. 1991;13(3):293-6.

54. Gustaffson PM, Tibbling L. Gastroesophageal reflux and oesophageal dysfunction in children and adolescents with brain damage. Acta Paediatr. 1994;83(10):1081-5.

55. Barrón F, Riquelme M, Elizondo J, Químbar A. Reflujo gastroesofágico y problemas respiratorios en parálisis cerebral infantil [monografía en Internet]. San Pedro Garza Garcia: Experiencia del Instituto Nuevo Amanecer [citado el 17 de enero de 2005]. Disponible en: http:// w w w. nu e voa manecer.edu. mx/medica/ invest_publicaciones.asp

56. Spiroglou K, Xinias I, Karatzas N, Karatza E, Arsos G, Panteliadis C. Gastric emptying in children with cerebral palsy and gastroesophageal reflux. Pediatr Neurol. 2004,31(3): 177-82.

57. Reyes AL, Cash AJ, Green SH, Booth IW. Gastroesophageal reflux in children with cerebral palsy. Child Care Health Dev. 1993;19(2):109-18.

58. Orenstein $\mathrm{S}$, Izadnia $\mathrm{F}$, Khan $\mathrm{S}$. Gastroesophageal reflux disease in children. Gastroenterol Clin North Am. 1999;28(4):947-69.

59. Jung AD. Gastroesophageal reflux in infants and children. American Family Physician. 2001;64(11):1853-60.

60. Sharma P, Sharma U, Kabra A. Cerebral Palsy - Clinical profile and predisposing factors. Indian Pediatrics. 1999;36:1038-42.

61. Krieger E, Hartl A, Scott K. Early childhood anemia and mild or moderate retardation. Am J Clin Nutr January. 1999;69(1):115-9.

62. Grantham-McGregor SM, Ani C. A review of studies on the effect of iron deficiency on cognitive development in children. J Nutr. 2001;131(suppl):649S-68S.

63. Pollitt E. Functional significance of the covariance between protein energy malnutrition and iron deficiency anemia. J Nutr. 1995;125:2272S-7S

64. Towkins A, Watson F. Malnutrition and infection. A review. London: Clinical Nutrition Unit. Centre for Human Nutrition. School of Hygiene and Tropical Medicine; 1989.

65. Solomon, Noel, Rosales. Parasitosis y nutrición. Cuaderno de Nutrición Nro. 3. Guatemala: Instituto de Nutrición de Centroamérica y Panamá; 1986.

66. Publicaciones del Instituto de Centroamérica y Panamá relacionados con nutrición e infección. Junio 1990.

67. Gupta M. Effect of ascariasis upon nutritional status of children. J Trop Pediatr. 1990;36:189-91.

Manuscrito recibido el 25 de mayo de 2006 y aceptado para publicación el 16 junio de 2006.

Correspondencia:

Dr. Aníbal Del Águila E.

Av. José Pardo 930 dpto. 202

Lima 18, Perú.

Correo-e: adelaguila80@yahoo.com 